\title{
Effect of single bout of passive stretching and Mulligan's Bent Leg Raise (BLR) on Hamstring flexibility in young adults with asymptomatic bilateral Hamstring tightness
}

\author{
Oves Patni ${ }^{1}$, Saravanan $\mathrm{M}^{2}$, Aliya Shaikh ${ }^{3}$, Ankita Juneja ${ }^{3}$, Nazrana Shaikh ${ }^{3}$, \\ Ruchi Patel ${ }^{3}$ \\ ${ }^{1}$ Lecturer, ${ }^{2}$ Senior Lecturer, ${ }^{3}$ Physiotherapist The Sarvajanik College of Physiotherapy, Badatwadi, Chhada- \\ Ole, Rampura, Surat - 395003 Gujarat, India
}

\begin{abstract}
:
Objectives: Various stretching techniques have been used to improve muscle flexibility in rehabilitation and sports. Mechanisms involving the efficacy of a stretching technique were studied and certain conclusions indicate one technique better than the other. Passive stretching is one most commonly used method and the recent inclusion of other techniques like ballistic stretching, Proprioceptive Neuromuscular Facilitation (PNF) techaniques with its variants and Mulligan's Bent Leg Raise (BLR) have created more scope for researchers to compare and conclude better methods for improving flexibility. The aim of the present study was to measure hamstrings flexibility after a single bout of passive hamstring muscle stretching and BLR and comparing the effects between the two techniques.

Methods: 78 asymptomatic individuals with bilateral hamstring tightness were randomized to one of the two groups - passive stretching $(n=39)$ and BLR $(n=39)$. Hamstring flexibility was measured using Back Saver Sit and Reach Test before and after the tests for both right and left lower limbs.

Results and Conclusion: Analysis using dependent revealed that both passive stretching and BLR groups improved hamstring flexibility on both sides with improvements of $3.7 \mathrm{~cm}$ and $4 \mathrm{~cm}$ on right and left side respectively in BLR group and $1.3 \mathrm{~cm}$ and $2 \mathrm{~cm}$ on right and left side respectively in passive stretching group. However results of between group comparisons using independent t tests were not significant ( $p=.992$ and .729 respectively for right and left sides) which suggests that passive stretching is equally effective as BLR technique in improving hamstrings length.
\end{abstract}

Keywords: Hamstring flexibility, Mulligan's Bent Leg Raise, passive stretching, Back Saver Sit and Reach test, passive knee extension test

\section{Introduction}

Flexibility is ability to move a single joint or series of joints through an unrestricted painful range of motion (ROM). It is dependent upon the extensibility of muscle which allows muscle that cross a joint to relax , lengthen and yield to a stretch force. Often the term flexibility is used to refer more specifically to the ability of musculotendinous unit to elongate as a body segment or when joint moves through ROM. Maintenance of flexibility is an essential component of any conditioning program in developing its mobility, improving athletic performance and preventing injuries. Good flexibility provides relaxation, posture eases muscular pain, helping quick recovery, reducing stress, keeps the body feel loose and agile ${ }^{1-3}$. As two- joint muscles, the ability to produce effective force at the knee is influenced by the relative position of the other joint over which that muscle crosses.

Greater hamstring force is produced with the hip in flexion when the hamstrings are lengthening over that joint regardless of the knee position ${ }^{4}$. When the two-joint hamstring are required to contract with hip extended and the knee flexed to 90degree or more, the hamstring must shorten over both the hip and knee. The hamstring will weaken as knee flexion proceed because not only are they approaching maximum shortening capability but also the muscle group must overcome the increasing tension in rectus femoris muscle that is approaching passive insufficiency ${ }^{4}$. Under ordinary condition two joint muscles are seldom used to move both joints simultaneously and more often the action of two joint muscles is prevented at one joint by resistance from gravity or continuation of other muscle, so a two joint muscle have tendency to shorten quickly. Running long distance causes the muscle that are active to become strong and less flexible where as opposing muscle which are relatively underused, becomes weaker. Immobilization for prolonged period also cause muscle tightness as the muscle is not used since longer time. Slow muscle fibers maintain posture; they activate more easily and are capable of more sustained contraction and tend to become shortened and tight. In female, wearing high heels for long period of time causes hamstrings tightness. 
Stretching is a form of physical exercise in which a specific skeletal muscle (or muscle group) is deliberately elongated to its fullest length (often by abduction from the torso) in order to improve the muscle's felt elasticity and reaffirm comfortable muscle tone. The result is a feeling of increased muscle control, flexibility and range of motion. Stretching is also used therapeutically to alleviate cramps. It increase muscle flexibility, maintain and augments the ROM and increase the elasticity and length of musculotendinous unit. It also permits the musculotendinous unit to store more energy in its viscoelastic \& contractile components ${ }^{5}$.

The common stretching technique used in rehabilitation is passive stretching administered by the therapist or the care giver ${ }^{6}$. Various studies in the past have recommended passive stretching to manage hamstrings tightness as it is safe to use and its effectiveness is well documented ${ }^{7}$. Studies on other stretching techniques like Proprioceptive Neuromuscular Facilitation $(\mathrm{PNF})^{7-9}$ or ballistic stretching in athletes also provide enough insight into the methods ${ }^{10}$ available for improving hamstring flexibility. BLR ${ }^{11}$ is a Mulligan stretching technique which is a recent advancement in the management of hamstrings tightness. It is a painless and advantageous technique, which is indicated to hamstring tightness with limitation of Straight Leg Raise (SLR). It can be tried even in the patients of low back pain who has limited or painful SLR. It can be tried even if they have leg pain above knee and it is extremely useful in the patients who have gross bilateral limitations of straight leg raise. Studies in the past have focused to compare effects of different types of stretching in overcoming hamstring tightness.

The present study focused on student population in the age group of 17 to 23 years as they have the posture of sitting with hip and knee flexion at $90^{\circ}$ which predisposes them to hamstring tightness. Also, scarce data is available on the immediate effects of stretching of any type after a short bout which if proven can be of highest significance to this population. Hence, we focused on evaluating students with bilateral hamstrings tightness and determining the effects of passive stretching and BLR on hamstring tightness in this study. We also extended our study to compare the effects of the above mentioned types of stretching before and after its application on both sides of the lower body.

\section{Methods}

Students of first, second, third and fourth year from Sarvajanik College of Physiotherapy were evaluated for hamstring flexibility. Out of 160 students, 78 were found to have bilateral hamstring tightness. Range of motion of knee extension with hip in $90^{\circ}$ flexion was measured passively through Passive Knee Extension test ${ }^{12}$ (PKE) using a goniometer. Participants who failed to extend the knee to full extension $\left(0^{0}\right)$ by $25^{0}$ or more were considered to have hamstring tightness ${ }^{13}$. Since none of these students had any recent neuromusculoskeletal deficits or injury and fit in the inclusion criteria, they were randomized to either BLR group or passive stretching group for experiment. Before and after either of the stretching technique was administered, Back Saver Sit and Reach test ${ }^{14}$ was used to measure the distance (in centimeters) reached which was recorded.

\subsection{Procedure}

\subsubsection{Bent Leg Raise (BLR) Technique}

BLR technique consist of gentle isometrics stretching of hamstring in specific directions in progressively greater positions of hip flexion, the expecting results are increased flexibility of hamstring muscle with increased ROM of active knee extension. The procedure for performing BLR ${ }^{11}$ was as follows:

Participant was in supine lying on a high couch with the investigator in walk stand position lateral to the leg, which was being stretched. Hip and Knee of the side to be stretched was bent at $90^{\circ}-90^{\circ}$. Investigator placed participant's flexed knee over his shoulder, the popliteal fossa of the knee resting on the his shoulder. A distraction (longitudinal traction force along the long axis of femur) was applied at the lower end of femur and the participant was asked to push the investigator's shoulder with his or her leg followed by voluntary relaxation.

At this point of relaxation, the investigator pushed the bent knee up as far as possible in the direction of the shoulder on the same side in a pain free range. This stretch was sustained for 5-10 seconds and then relaxed. If the pain or restriction eased, the hip was taken further in to flexion. It was ensured that there was no pain during the procedure, if it was painful the direction of the leg raise was altered medially or laterally. The process was repeated till the knee of the participant was beyond the shoulder of therapist. The contra lateral leg was kept relaxed and allowed to move as it goes. At the end of the range, the position was held for 10 seconds and limb brought back to the neutral position. The traction was maintained throughout the technique.

\subsubsection{Passive Stretching Technique}

Participants were positioned in supine lying with low back as flat as possible. Hip was flexed to $90^{\circ}$ with the slightly flexed knee supported by the investigator at the ankle by placing it on his shoulder. The opposite extremity was stabilized along the anterior aspect of thigh with the support of assistance of other person. With the knee in maximum extension, the hip was flexed as far as possible until the participant felt the 
stretch feeling on the posterior thigh ${ }^{10}$. The position of sub maximal stretch was maintained for a hold of 10 seconds.

\subsubsection{Back-Saver Sit and Reach Test}

The back-saver sit and reach is very similar to the traditional sit and reach except that the measurement is performed on one side at a time. By testing one leg at a time a determination can be made of any asymmetry in hamstring flexibility, and hyperextension of both knees is avoided ${ }^{15}$. The sit and reach measures predominantly the flexibility of the hamstring muscles. Normal hamstring flexibility allows rotation of the pelvis in forward bending movements and posterior tilting of the pelvis for proper sitting. The back-saver sit and reach has been shown to provide extremely consistent scores when administered under standardized conditions ${ }^{16}$. The back-saver sit and reach has also been shown to be a reasonably accurate measure of hamstring flexibility. This test involved sitting on the floor with legs without shoes stretched out straight ahead.

The soles of the feet were placed flat against a custom made sit and reach box. Both knees were locked and pressed flat to the floor. With the palms facing downwards, and the hands on top of each other, the participant reached forward along the measuring line as far as possible ensuring that the hands remain at the same level, not one reaching further forward than the other. The participant reached out and held that position for one-two seconds while the distance was recorded. It was instructed and made sure that there are no jerky movements. The score was recorded to the nearest centimeter as the distance reached by the hand.

\subsubsection{Data analysis}

Descriptive statistics (mean and standard deviation), dependent and independent $t$ test were used at $p<0.05$ level of significance. Dependent $t$ test was employed for comparing means of pre and post test differences within groups and independent $t$ test for between the two groups. SPSS v 20.0 was used for analysis of data.

\section{Results}

Analyses were made in three steps to statistically find out the differences in scores of hamstring flexibility. Dependent $t$ test was used separately for each group to observe the changes in the distance covered pre and post Back Saver Sit and Reach test. Independent $t$ test was employed to observe the differences between pre test and posttest BLR and passive stretching groups.

TABLE 1 represents the mean scores of pre test and posttest values in centimeters for right and left side legs of BLR and passive stretching groups. It also indicates the difference in means of each groups on both sides. Dependent $t$ test for BLR group showed significant differences pre and post application of BLR on both right and left sides $(\mathrm{df}=38, \mathrm{t}=-9.896 \mathrm{p}<0.000)$ and $(\mathrm{df}=38, \mathrm{t}=-10.817 \mathrm{p}<0.000)$ respectively. Similarly, significant differences were observed when comparisons were made between pre and posttest scores for right and left side in passive stretching group with $\mathrm{df}=38, \mathrm{t}=-6.047 \mathrm{p}<0.000$ and $\mathrm{df}=38, \mathrm{t}=-10.720 \mathrm{p}<0.000$ respectively.

Difference in gain of hamstring flexibility in BLR group was $3.7 \mathrm{~cm}$ on right side and $4 \mathrm{~cm}$ on left side (TABLE $1 \&$ Fig 1). The gain was slightly lower in passive stretching group with $1.3 \mathrm{~cm}$ on right and $2 \mathrm{~cm}$ on left showing better gain in flexibility in BLR group. Left side gains were comparatively better than right side gains in both groups. However, separate analysis using independent $t$ test of pretest and posttest comparisons for both right and left sides between groups indicated no significant difference (Fig 1). Pretest results of independent $\mathrm{t}$ test for the right side and left side were $\mathrm{t}=-1.736, \mathrm{p}=.087, \mathrm{t}=-1.190, \mathrm{p}=.238$ respectively. Posttest results of independent $\mathrm{t}$ test for the right side and left side were $\mathrm{t}=0.10, \mathrm{p}=.992, \mathrm{t}=.348, \mathrm{p}=.729$ respectively.

IV. Tables And Figures

Table 1: Descriptive statistics [Mean (in $\mathrm{cm}) \pm \mathrm{SD}$ ] of right and left side, pre and post stretching of both groups

\begin{tabular}{|c|c|c|c|c|c|c|c|c|}
\hline & \multicolumn{8}{|c|}{ GROUPS } \\
\hline & \multicolumn{4}{|c|}{$\operatorname{BLR}(n=39)$} & \multicolumn{4}{|c|}{ PS $(n=39)$} \\
\hline & \multicolumn{2}{|c|}{ Right } & \multicolumn{2}{|c|}{ Left } & \multicolumn{2}{|c|}{ Right } & \multicolumn{2}{|c|}{ Left } \\
\hline & Mean & SD & Mean & SD & Mean & SD & Mean & SD \\
\hline Pretest & 21.46 & 5.83 & 21.41 & 5.50 & 23.83 & 6.24 & 22.99 & 6.18 \\
\hline Posttest & 25.15 & 5.63 & 25.46 & 5.51 & 25.14 & 5.99 & 25.00 & 6.17 \\
\hline Difference & 3.69 & -.20 & 4.05 & .01 & 1.31 & -.25 & 2.01 & -.01 \\
\hline
\end{tabular}

BLR - Bent leg raise

PS - Passive stretching 


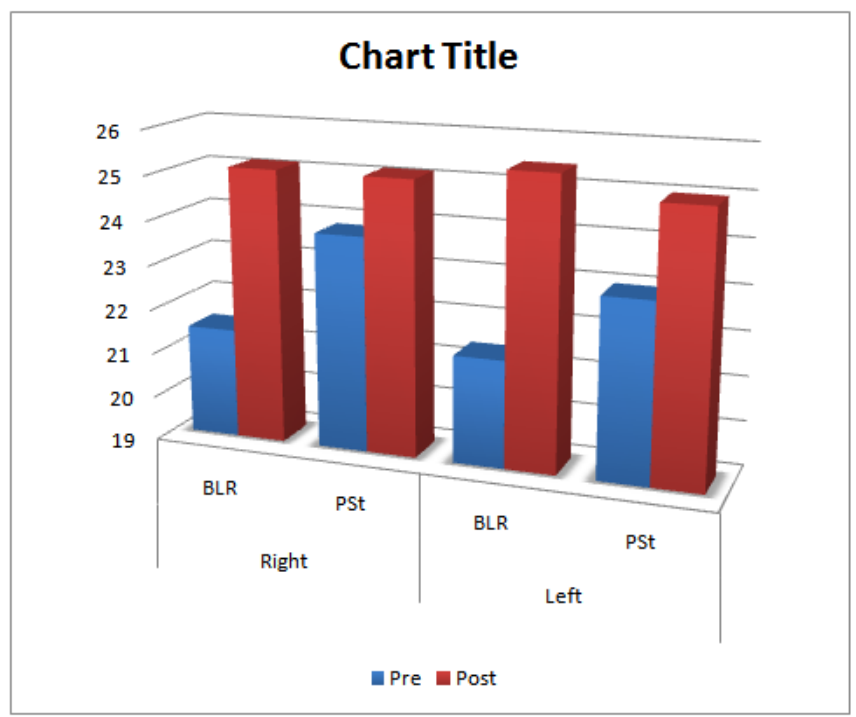

Figure 1: Mean comparison of pre and post stretching for right and left side between BLR and Passive stretching

\section{Discussion}

Passive stretching has been emphasized as a technique effective in improving flexibility of muscles especially in hamstrings. The introduction of other methods in affecting muscle extensibility in the recent past has increased the interest of researchers to find out better techniques to treat muscle tightness. Bent leg raise (BLR) is one such technique which is being widely used in practice but has been least studied on its effectiveness to reduce tightness of hamstrings muscles.

The present study compared the effectiveness of a single bout of BLR and passive stretching in asymptomatic college students with bilateral hamstring tightness. Even though BLR produced a change of 3.7 and $4 \mathrm{cms}$ difference in pre and post test measures when observed within groups, a comparison between groups failed to produce significant differences which shows that BLR does not have any clinical advantage than passive stretching in improving hamstring flexibility. Previous studies by Halbertsma et $\mathrm{al}^{6}$ (1999) concluded that the acute effects of repeated passive stretching is negligible which is supported by the results of our study.

In contrary, Volkert et al ${ }^{17}$ (2003) found significant increase $\left(13^{0}-14^{0}\right.$ as measured using active knee extension) in hamstrings length post static stretching. Toby Hall et $\mathrm{al}^{11}$ (2005) concluded that after a single intervention of Mulligan's BLR technique, immediate improvement were not observed but the technique was effective in improving the range of straight leg raise (SLR) after 24 hours. They also added that BLR technique was no better than placebo. Since studies on BLR are scarce and highly variable, the results of the present study can be of significance in setting a baseline for further future studies.

\section{Conclusion}

In young adults with asymptomatic bilateral hamstring tightness, hamstring flexibility gains obtained from a single bout of both passive stretching and BLR were almost similar. The present study concludes that both passive stretching and BLR interventions can significantly improve hamstrings flexibility and the difference between the changes produced by both the interventions are negligible.

\section{References}

[1]. Wiemann K, Hahn K. Influences of strength, stretching and circulatory exercises on flexibility parameters of the human hamstrings. Int J Sports Med 1997;18(5):340-6.

[2]. Halbertsma JP, Goeken LN. Stretching exercises: effect on passive extensibility and stiffness in short hamstrings of healthy subjects. Arch Phys Med Rehabil 1994;75(9):976-81.

[3]. Matsuo S, Suzuki S, Iwata M, Banno Y, Asai Y, Tsuchida W, et al. Acute effects of different stretching durations on passive torque, mobility, and isometric muscle force. J Strength Cond Res 2013.

[4]. Costa PB, Ryan ED, Herda TJ, Walter AA, Defreitas JM, Stout JR, et al. Acute effects of static stretching on peak torque and the hamstrings-to-quadriceps conventional and functional ratios. Scand J Med Sci Sports 2013;23(1):38-45.

[5]. Halbertsma JP, van Bolhuis AI, Goeken LN. Sport stretching: effect on passive muscle stiffness of short hamstrings. Arch Phys Med Rehabil 1996;77(7):688-92.

[6]. Halbertsma JP, Mulder I, Goeken LN, Eisma WH. Repeated passive stretching: acute effect on the passive muscle moment and extensibility of short hamstrings. Arch Phys Med Rehabil 1999;80(4):407-14.

[7]. O'Hora J, Cartwright A, Wade CD, Hough AD, Shum GL. Efficacy of static stretching and proprioceptive neuromuscular facilitation stretch on hamstrings length after a single session. J Strength Cond Res 2011;25(6):1586-91.

[8]. Streepey JW, Mock MJ, Riskowski JL, Vanwye WR, Vitvitskiy BM, Mikesky AE. Effects of quadriceps and hamstrings proprioceptive neuromuscular facilitation stretching on knee movement sensation. J Strength Cond Res 2010;24(4):1037-42. 
[9]. Nordez A, McNair P, Casari P, Cornu C. Acute changes in hamstrings musculo-articular dissipative properties induced by cyclic and static stretching. Int J Sports Med 2008;29(5):414-8.

[10]. Chan SP, Hong Y, Robinson PD. Flexibility and passive resistance of the hamstrings of young adults using two different static stretching protocols. Scand J Med Sci Sports 2001;11(2):81-6.

[11]. Hall T, Hardt S, Schafer A, Wallin L. Mulligan bent leg raise technique--a preliminary randomized trial of immediate effects after a single intervention. Man Ther 2006;11(2):130-5.

[12]. Guex K, Fourchet F, Loepelt H, Millet GP. Passive knee-extension test to measure hamstring tightness: influence of gravity correction. J Sport Rehabil 2012;21(3):231-4.

[13]. Marques AP, Vasconcelos AA, Cabral CM, Sacco IC. Effect of frequency of static stretching on flexibility, hamstring tightness and electromyographic activity. Braz J Med Biol Res 2009;42(10):949-53.

[14]. Chillon P, Castro-Pinero J, Ruiz JR, Soto VM, Carbonell-Baeza A, Dafos J, et al. Hip flexibility is the main determinant of the back-saver sit-and-reach test in adolescents. J Sports Sci 2010;28(6):641-8.

[15]. Hui SS, Yuen PY. Validity of the modified back-saver sit-and-reach test: a comparison with other protocols. Med Sci Sports Exerc 2000;32(9):1655-9.

[16]. Patterson P, Wiksten DL, Ray L, Flanders C, Sanphy D. The validity and reliability of the back saver sit-and-reach test in middle school girls and boys. Res Q Exerc Sport 1996;67(4):448-51.

[17]. Volkert C. de Weijer GCG, Eric Shamus. The Effect of Static Stretch and Warm-up Exercise on Hamstring Length Over the Course of 24 Hours. J Orthop Sports Phys Ther 2003;33:727-33. 